\title{
The prognostic value of right ventricular longitudinal strain and 3D ejection fraction in patients with dilated cardiomyopathy
}

\author{
Aura Vîjîiac $^{1,2} \cdot$ Sebastian Onciul ${ }^{1,2} \cdot$ Claudia Guzu $^{2} \cdot$ Violeta Verinceanu $^{2} \cdot$ Vlad Bătăilă $^{2} \cdot$ Silvia Deaconu $^{1,2}$. \\ Alina Scărlătescu ${ }^{2} \cdot$ Diana Zamfir $^{2} \cdot$ Ioana Petre $^{1,2} \cdot$ Roxana Onuț $^{2} \cdot$ Alexandru Scafa-Udriste $^{1,2} \cdot$ Radu Vătășescu $^{1,2}$ (1) \\ Maria Dorobanțu ${ }^{1,2}$
}

Received: 28 April 2021 / Accepted: 18 June 2021 / Published online: 24 June 2021

(c) The Author(s), under exclusive licence to Springer Nature B.V. 2021

\begin{abstract}
Several studies showed that right ventricular (RV) dysfunction is a powerful predictor in heart failure (HF). Advanced echocardiographic techniques such as speckle-tracking imaging and three-dimensional (3D) echocardiography proved to be accurate tools for RV assessment, but their clinical significance remains to be clarified. The aim of this study was to evaluate the role of two-dimensional (2D) RV strain and 3D ejection fraction (RVEF) in predicting adverse outcome in patients with non-ischemic dilated cardiomyopathy (DCM). We prospectively screened 81 patients with DCM and sinus rhythm, 50 of whom were enrolled and underwent comprehensive echocardiography, including RV strain and 3D RV volumetric assessment. Patients were followed for a composite endpoint of cardiac death, nonfatal cardiac arrest and acute worsening of HF requiring hospitalization. After a median follow-up of 16 months, 29 patients reached the primary endpoint. Patients with events had more impaired RV global longitudinal strain $(-10.5 \pm 4.5 \%$ vs. $-14.3 \pm 5.2 \%, \mathrm{p}=0.009)$, RV free wall longitudinal strain $(-12.9 \pm 8.7 \%$ vs. $-17.5 \pm 7.1 \%, p=0.046)$ and 3 D RVEF $(38 \pm 8 \%$ vs. $47 \pm 9 \%, p=0.001)$. By Cox proportional hazards multivariable analysis, RV global longitudinal strain and RVEF were independent predictors of outcome after adjustment for age and NYHA class. RVEF remained the only independent predictor of events after further correction for echocardiographic risk factors. By receiver-operating characteristic analysis, the optimal RVEF cut-off value for event prediction was $43.4 \%$ (area under the curve $=0.768, \mathrm{p}=0.001$ ). Subjects with $\mathrm{RVEF}>43.4 \%$ showed more favourable outcome compared to those with RVEF $<43.4 \%$ (log-rank test, $\mathrm{p}<0.001$ ). In conclusion, 3D RVEF is an independent predictor of major adverse cardiovascular events in patients with DCM.
\end{abstract}

Keywords Dilated cardiomyopathy $\cdot$ Right ventricular strain $\cdot$ Right ventricular ejection fraction

\section{Introduction}

Patients with dilated cardiomyopathy (DCM) represent a heterogenous group in terms of both morpho-functional characteristics of the left and right heart chambers and of clinical outcome. While most of the research focused on the left ventricular (LV) function, establishing a well-known prognostic role for LV ejection fraction (LVEF) in DCM [1, 2], during the last decade several studies raised awareness of

Radu Vătăşescu

radu_vatasescu@yahoo.com

1 "Carol Davila" University of Medicine and Pharmacy, Bucharest, Romania

2 Emergency Clinical Hospital, 8, Calea Floreasca, 014491 Bucharest, Romania the importance of right ventricular (RV) dysfunction in the setting of both right and left heart disease [3-6].

However, the complex anatomy and asymmetrical shape of the RV make its echocardiographic assessment challenging, usually requiring a multi-parametric approach [7]. While cardiac magnetic resonance (CMR) remains the goldstandard for the evaluation of RV volumes and function [8], it is not a tool for routine assessment of cardiac patients. Modern techniques such as speckle-tracking echocardiography (STE) and three-dimensional (3D) echocardiography have been validated against CMR $[9,10]$, but the prognostic role of RV strain indices and 3D RV functional parameters in DCM remains to be clarified.

Therefore, the aims of this study were: (1) to evaluate the extent of RV dysfunction-assessed by modern echocardiography techniques-in a cohort of patients with DCM and 
(2) to evaluate the ability of RV strain and 3D RV ejection fraction (RVEF) to predict major adverse events in these patients.

\section{Materials and methods}

\section{Study population}

Consecutive patients with DCM and sinus rhythm were enrolled in a prospective single-centre study between January 2019 and January 2020. The diagnostic of DCM [11] required all 3 following criteria to be met: (1) dilated LV, according to cut-offs from the current guidelines of chamber quantification [12]; (2) LVEF $<40 \%$ by two-dimensional (2D) Simpson biplane method and (3) absence of significant coronary artery disease (defined as $>50 \%$ stenosis of a major epicardial vessel). Exclusion criteria were poor acoustic window, inability to hold breath, other comorbidities with life expectancy $<1$-year and cor pulmonale.

Basic demographic and clinical data, past medical history and blood test results were collected by three investigators. All patients received optimal pharmacological therapy according to heart failure (HF) guidelines [2], including biventricular pacing when indicated. Loop diuretics were administered if congestion was present, and their dose was adjusted according to the patients' symptoms. The study protocol complied with the Declaration of Helsinki and it was approved by the human research committee of our hospital.

\section{Two-dimensional echocardiography}

Comprehensive 2D echocardiographic examinations were performed at enrolment using a Vivid E9 (GE Vingmed, Horten, Norway) ultrasound machine, according to current recommendations [13]. Patients had been clinically stable at the time of the examination for at least 3 days, with no significant change in medication or hemodynamic status and no need for catecholamine use. Three independent researchers blinded to follow-up clinical data performed offline data analysis using dedicated software (EchoPAC BT 12).

$\mathrm{RV}$ systolic function was assessed both by conventional parameters such as tricuspid annular plane systolic excursion (TAPSE), pulsed tissue Doppler $S$ wave velocity, RV fractional area change (RV-FAC) and by special parameters such as global longitudinal strain (GLS) of the RV and strain of the RV free wall (RVFW). All conventional parameters were measured according to current international recommendations [14]. Pulmonary artery systolic pressure (PASP) was assessed using the gradient between the RV and the right atrium (RA) - obtained from the tricuspid regurgitation (TR) jet-and the estimated RA pressure, based on the diameter and collapsibility of the inferior vena cava.

For strain analysis we used 2D strain software (EchoPAC-Q Analysis package) using high frame rate acquisitions (50-70 frames per second). The endocardial border of the RV was manually traced from the apical RVfocused view, and the width of the speckle-tracking region of interest was adjusted to include the whole myocardial wall but to exclude the pericardium, as recommended [15, 16]. Both the RVFW and the interventricular septum (IVS) were automatically divided into three segments. Global longitudinal strain of the RV (GLS-RV) was calculated as the mean of the six segmental values, while the longitudinal strain of the RVFW (RVFW-LS) was calculated as the average of the three segmental values of the RVFW (Fig. 1). Since strain is a measure of myocardial shortening, normal strain values are negative [15], with higher strain values (less negative) unravelling impaired longitudinal shortening.

\section{Three-dimensional echocardiography}

Six-beat full-volume 3D data sets were acquired with a $4 \mathrm{~V}$ probe in all patients. $\mathrm{RV}$-focused $3 \mathrm{D}$ data sets were obtained from the apical RV-focused view, with electrocardiographic gating during breath holding. Image analysis was performed offline using commercially available software: 4D AutoLVQ (EchoPAC BT 12, GE Vingmed-Ultrasound, Horten, Norway) for the LV volumes and LVEF and 4D RV-Function (TomTec Imaging Systems GmbH, Unterschleissheim, Germany) for the RV volumes and RVEF.

For RV assessment, the basal and apical RV transversal axes were set, and the operator selected landmarks corresponding to centre of the tricuspid and the mitral valve. The software provided the RV apical, sagittal and coronal views and the RV endocardial border was manually traced in endsystole and end-diastole, including the trabeculations and moderator band within the RV cavity [17]. Finally, the software provided the 3D RV end-diastolic volume, end-systolic volume, stroke volume and RVEF (Fig. 2).

\section{Reproducibility}

To test the intraobserver reproducibility of RV strain and 3D RVEF, measurements were repeated two weeks apart in 10 randomly selected patients. To test the interobserver variability, the same 10 patients were measured by a second researcher, blinded to the prior measurements. Reproducibility was assessed by calculating the intraclass coefficient (ICC) on a two-way mixed-effects model. 


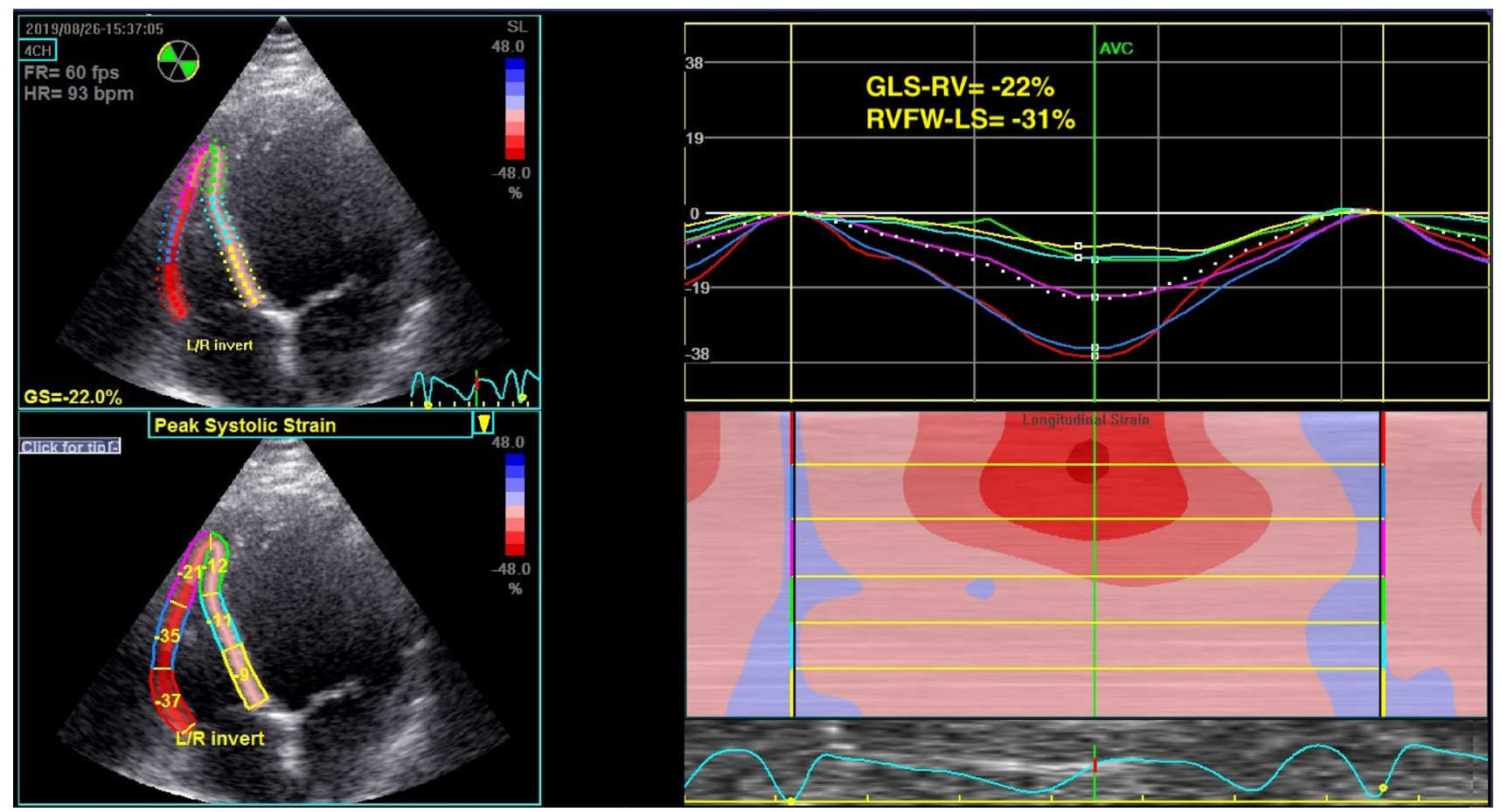

Fig. 1 Illustration of right ventricular strain measurement using speckle-tracking echocardiography. $G L S$ - $R V$ global longitudinal strain of the RV, $R V F W-L S$ RV free wall longitudinal strain

\section{Follow-up}

Patients were prospectively followed to ascertain the occurrence of any major adverse cardiovascular event (MACE). For the current study, the primary endpoint was the composite of cardiac death, nonfatal cardiac arrest and HF exacerbation requiring hospitalization. Cardiac death was defined as death resulting from an acute coronary syndrome, fatal arrythmia, acute worsening of HF and sudden cardiac death. For patients without MACE, survival analysis was performed using the date of the last contact. Follow-up was conducted for $16 \pm 3$ months.

\section{Statistical analysis}

All statistical analysis was performed using SPSS version 20.0 statistical software package. The normal distribution of variables was assessed by Kolmogorov-Smirnov test. Continuous data were summarized as mean \pm standard deviation if normally distributed and as median and interquartile range otherwise. To compare continuous data, we used Student's $\mathrm{t}$ test (for normally distributed variables) or Mann-Whitney $\mathrm{U}$ test (for non-normally distributed variables). Categorical data were displayed as numbers and percentages and they were compared using $\chi^{2}$ test or Fisher exact test, as appropriate. Correlations between continuous variables were assessed using Pearson's correlation coefficient.
Time-dependent receiver operating characteristic (ROC) curves and the respective area under the curve (AUC) were used to assess the ability of RV functional parameters to predict adverse outcome. Cut-off values for each parameter were chosen based on the highest sum of sensitivity and specificity. Event-free survival was assessed using Kaplan-Meier analysis and survival curves were compared with the log-rank test. We performed Cox proportional hazards univariable analysis in order to determine significant predictors for MACE. Results were reported as hazard ratios (HR) with $95 \%$ confidence intervals (CI). Variables with statistical significance in univariable analysis were further incorporated a multivariable Cox proportional-hazards regression, which also included age-regardless of its significance in univariable analysis. P-values $<0.05$ were considered statistically significant.

\section{Results}

\section{Baseline characteristics}

Of the 81 eligible patients initially screened, $14(17 \%)$ had an acoustic window unsuitable for either strain or 3D analysis, $4(5 \%)$ had cor pulmonale and $13(16 \%)$ were lost to follow-up, leaving a final study population of 50 patients. Mean 
Fig. 2 Three-dimensional assessment of the right ventricle using dedicated software. ESV end-systolic volume, $E D V$ enddiastolic volume, $E F$ ejection fraction, $S V$ stroke volume

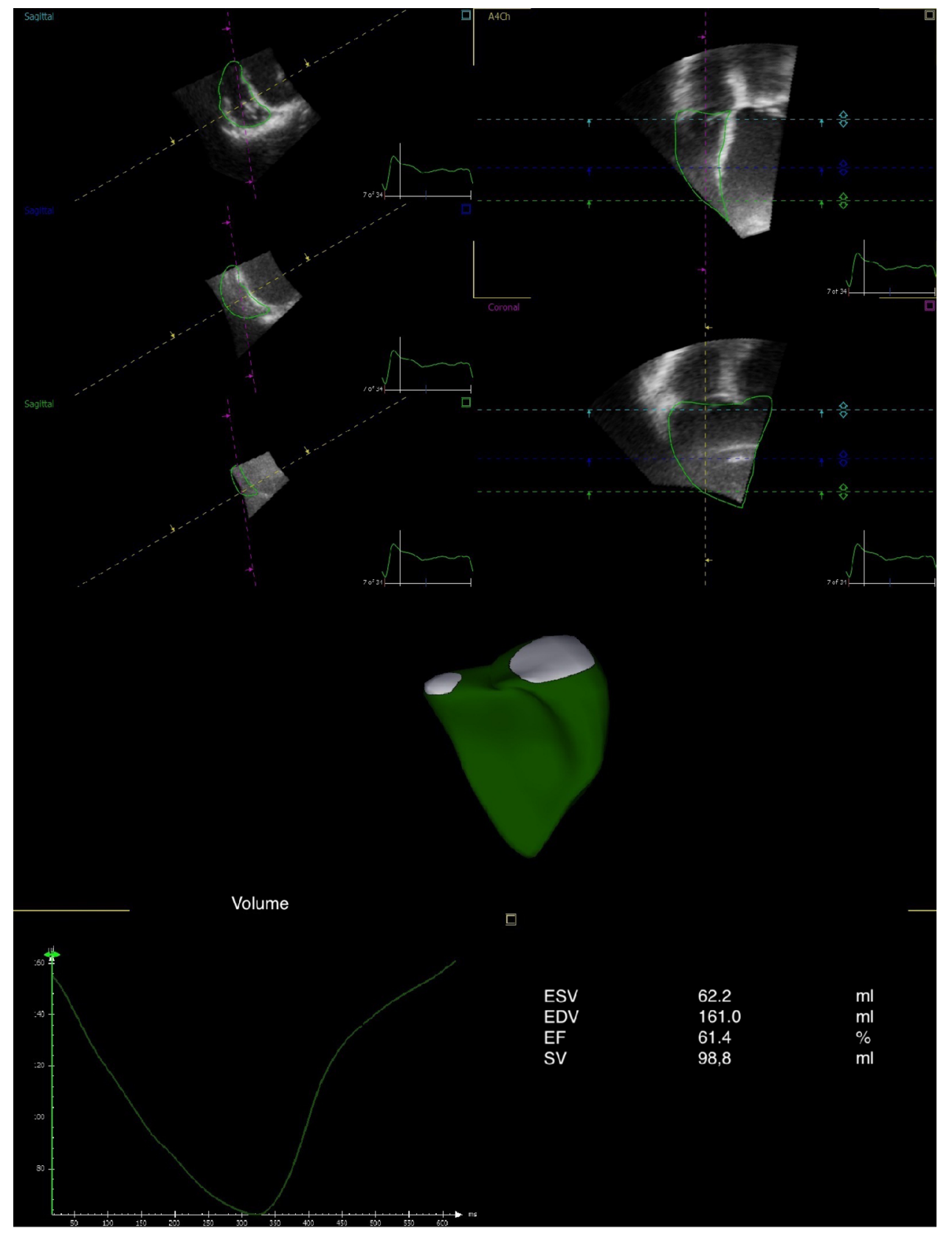

age was $61 \pm 14$ years and the majority were men $(68 \%)$. The cohort encompassed both newly- and previously diagnosed DCM patients, with a median time since first diagnostic of 9 (1-36) months. During the mean follow-up of $16 \pm 3$ months, 29 patients $(58 \%)$ reached the primary end point: there were 3 cardiac deaths (6\%), 3 nonfatal cardiac arrests $(6 \%)$ and 23 readmissions (46\%) for HF exacerbation. Baseline demographic and clinical characteristics are summarised in Table 1.

\section{D echocardiographic findings}

Mean LVEF in the study group was $25 \pm 7 \%$. There were no significant differences between the two subgroups in terms of LV dimensions and systolic function. Patients in the MACE subgroup had lower A wave velocity, higher mitral $\mathrm{E} / \mathrm{E}^{\prime}$ ratio and higher left atrial (LA) volume index, reflecting a more severe diastolic dysfunction. PASP did not differ significantly between the two subgroups. Baseline 2D echocardiographic characteristics are summarised in Table 2.

Both TAPSE and S wave velocity were significantly lower in patients with events. Based on literature cut-offs [12] for conventional parameters, the prevalence of $\mathrm{RV}$ dysfunction 
Table 1 Baseline clinical characteristics

\begin{tabular}{lllll}
\hline Variables & $\begin{array}{l}\text { All patients } \\
(\mathrm{n}=50)\end{array}$ & $\begin{array}{l}\text { MACE } \\
(\mathrm{n}=29)\end{array}$ & $\begin{array}{l}\text { No MACE } \\
(\mathrm{n}=21)\end{array}$ & P-value \\
\hline Age (years) & $61 \pm 14$ & $61 \pm 14$ & $60 \pm 15$ & 0.74 \\
Men, $\mathrm{n}(\%)$ & $34(68 \%)$ & $21(72 \%)$ & $13(62 \%)$ & 0.43 \\
Systolic BP (mm Hg) & $124 \pm 13$ & $121 \pm 13$ & $127 \pm 13$ & 0.16 \\
Diastolic BP (mm Hg) & $75 \pm 11$ & $72 \pm 10$ & $78 \pm 12$ & $\mathbf{0 . 0 4 6}$ \\
Heart rate (bpm) & $79 \pm 16$ & $79 \pm 18$ & $79 \pm 13$ & 0.94 \\
NYHA class III or IV, n (\%) & $26(52 \%)$ & $20(69 \%)$ & $6(28 \%)$ & $\mathbf{0 . 0 0 9}$ \\
Comorbidities, n (\%) & & & & \\
Hypertension & $34(68 \%)$ & $19(66 \%)$ & $15(71 \%)$ & 0.66 \\
Diabetes mellitus & $9(18 \%)$ & $7(24 \%)$ & $2(10 \%)$ & 0.18 \\
Smoking, $(\%)$ & $17(34 \%)$ & $10(35 \%)$ & $7(33 \%)$ & 0.93 \\
Medication, $\mathrm{n}(\%)$ & & & & \\
ACE-I/ARBs & $44(88 \%)$ & $24(83 \%)$ & $20(95 \%)$ & 0.18 \\
Beta-blocker & $49(98 \%)$ & $28(97 \%)$ & $21(100 \%)$ & 0.39 \\
MRA & $49(98 \%)$ & $28(97 \%)$ & $21(100 \%)$ & 0.39 \\
Loop diuretic & $34(68 \%)$ & $24(83 \%)$ & $10(48 \%)$ & $\mathbf{0 . 0 1}$ \\
Digoxin & $9(18 \%)$ & $8(28 \%)$ & $1(5 \%)$ & $\mathbf{0 . 0 4}$ \\
CRT, n (\%) & $19(37 \%)$ & $9(31 \%)$ & $10(48 \%)$ & 0.23 \\
\hline R & & &
\end{tabular}

Bolded p-values are statistically significant $<0.05$

Continuous data are expressed as mean \pm standard deviation. Categorical data are expressed as number (percentage)

$n$ number of patients, $M A C E$ major adverse cardiac events, $B P$ blood pressure, NYHA New York Heart Association, $A C E-I$ angiotensin-converting enzyme inhibitor, $A R B$ angiotensin receptor blocker, $M R A$ mineralocorticoid receptor antagonist, $C R T$ cardiac resynchronization therapy ranged between 34 and $54 \%$ in the entire study population. Mean GLS-RV in the study group was $-12.1 \pm 5.1 \%$, while mean RVFW-LS was $-14.8 \pm 8.3 \%$. Both GLS-RV and RVFW-LS were significantly more impaired in the MACE subgroup. Based on the normal threshold of $-20 \%$ for RVFW-LS recommended by current guidelines [12], $66 \%$ of all patients were classified as having RV dysfunction. Both GLS-RV and RVFW-LS were positively correlated with New York Heart Association (NYHA) class $(r=0.46$, $\mathrm{p}<0.001$ and $\mathrm{r}=0.40, \mathrm{p}=0.004$, respectively). GLS-RV and RVFW-LS had no correlation with either the degree of TR $(p=0.18$ and $p=0.30$, respectively) or with $\operatorname{PASP}(p=0.45$ and $\mathrm{p}=0.59$, respectively). Good intra- and interobserver reproducibility were found for GLS-RV (ICC $=0.91[95 \%$ CI, 0.64-0.98] and ICC $=0.88$ [95\% CI, 0.53-0.97], respectively) and for RVFW-LS (ICC $=0.87$ [95\% CI, 0.55-0.97] and $\mathrm{ICC}=0.82$ [95\% CI, 0.25-0.95], respectively).

\section{D echocardiographic findings}

3D echocardiographic data are presented in Table 3. 3D LVEF showed an excellent correlation with 2D LVEF $(\mathrm{r}=0.83, \mathrm{p}<0.001)$. Mean 3D RVEF in the study group was $42 \pm 10 \%$. The prevalence of RV systolic dysfunction assessed by 3D RVEF was $62 \%$, according to the threshold of $45 \%$ proposed by current guidelines [12] (Fig. 3). Good intra- and interobserver reproducibility were found for 3D RVEF (ICC $=0.93$ [95\% CI, 0.87-0.96] and ICC $=0.90[95 \%$ CI, 0.54-0.97], respectively). There were no significant differences between patients with preserved RVEF and patients with reduced RVEF in terms of age $(p=0.28), 2 \mathrm{D}$ LVEF $(p=0.37)$ or mitral $E / E^{\prime}$ ratio $(p=0.21)$; however, patients with reduced RVEF showed larger indexed LA volume $(52 \pm 28 \mathrm{ml}$ vs. $37 \pm 14 \mathrm{ml}, \mathrm{p}=0.01) . \mathrm{RVEF}$ had a negative correlation with GLS-RV $(r=-0.44, p=0.001)$, RVFWLS $(r=-0.42, p=0.002)$ and NYHA class $(r=-0.43$, $\mathrm{p}=0.002)$. No correlation was found between $3 \mathrm{D}$ RVEF and either 3D LVEF ( $p=0.17)$, PASP $(p=0.66)$, degree of TR $(p=0.40)$ or time since DCM diagnostic $(p=0.41)$.

\section{The prognostic role of RV function}

We performed ROC analysis to evaluate the association between RV functional parameters and outcome and to determine cut-offs with maximised sensitivity plus specificity (Fig. 4). TAPSE, S wave velocity, GLS-RV and 3D RVEF were good predictors of MACE, with TAPSE and 3D RVEF showing the best AUC (Table 4). The best cut-off for MACE prediction was $17.5 \mathrm{~mm}$ for TAPSE, $10.5 \mathrm{~cm} / \mathrm{s}$ for $\mathrm{S}^{\prime}$ wave velocity, $-12.5 \%$ for GLS-RV and $43.4 \%$ for RVEF. In Kaplan-Meier analysis, lower TAPSE, lower S wave velocity, more impaired GLS-RV and worse RVEF 
Table 2 Baseline 2D echocardiographic data

\begin{tabular}{|c|c|c|c|c|}
\hline Variables & $\begin{array}{l}\text { All patients } \\
(\mathrm{n}=50)\end{array}$ & $\begin{array}{l}\text { MACE } \\
(n=29)\end{array}$ & $\begin{array}{l}\text { No MACE } \\
(\mathrm{n}=21)\end{array}$ & P-value \\
\hline \multicolumn{5}{|l|}{ LV functional parameters } \\
\hline LVEDV index $\left(\mathrm{ml} / \mathrm{m}^{2}\right)$ & $119 \pm 42$ & $123 \pm 41$ & $113 \pm 45$ & 0.45 \\
\hline LVESV index $\left(\mathrm{ml} / \mathrm{m}^{2}\right)$ & $90 \pm 38$ & $94 \pm 37$ & $85 \pm 40$ & 0.47 \\
\hline $\operatorname{LVEF}(\%)$ & $25 \pm 7$ & $25 \pm 8$ & $26 \pm 7$ & 0.53 \\
\hline \multicolumn{5}{|l|}{ Transmitral flow parameters } \\
\hline E wave velocity $(\mathrm{cm} / \mathrm{s})$ & $74 \pm 24$ & $78 \pm 23$ & $69 \pm 24$ & 0.18 \\
\hline E wave DT (ms) & $169 \pm 58$ & $169 \pm 62$ & $168 \pm 53$ & 0.93 \\
\hline A wave velocity $(\mathrm{cm} / \mathrm{s})$ & $66 \pm 26$ & $60 \pm 24$ & $75 \pm 27$ & 0.04 \\
\hline $\mathrm{E} / \mathrm{A}$ ratio & $1.37 \pm 0.85$ & $1.52 \pm 0.72$ & $1.17 \pm 0.99$ & 0.48 \\
\hline Average $\mathrm{E} / \mathrm{E}^{\prime}$ ratio & $14.5 \pm 6.9$ & $16.4 \pm 7.8$ & $11.9 \pm 4.5$ & 0.01 \\
\hline GLS-LV $(\%)$ & $-7.4 \pm 2.8$ & $-7.2 \pm 3.3$ & $-7.8 \pm 2.0$ & 0.47 \\
\hline LA volume index $\left(\mathrm{ml} / \mathrm{m}^{2}\right)$ & $46 \pm 24$ & $53 \pm 27$ & $36 \pm 16$ & 0.008 \\
\hline \multicolumn{5}{|l|}{ RV functional parameters } \\
\hline RV basal diameter (mm) & $38 \pm 7$ & $40 \pm 8$ & $35 \pm 5$ & 0.02 \\
\hline RV longitudinal diameter (mm) & $68 \pm 11$ & $68 \pm 11$ & $69 \pm 11$ & 0.66 \\
\hline TAPSE (mm) & $18 \pm 5$ & $16 \pm 4$ & $21 \pm 4$ & 0.0004 \\
\hline $\mathrm{S}$ wave velocity $(\mathrm{cm} / \mathrm{s})$ & $10.7 \pm 2.4$ & $9.8 \pm 2.4$ & $11.9 \pm 2.0$ & 0.002 \\
\hline RV-FAC $(\%)$ & $33 \pm 12$ & $31 \pm 12$ & $35 \pm 12$ & 0.20 \\
\hline \multicolumn{5}{|l|}{ Tricuspid flow parameters } \\
\hline E wave velocity $(\mathrm{cm} / \mathrm{s})$ & $51 \pm 10$ & $53 \pm 10$ & $48 \pm 10$ & 0.08 \\
\hline E wave DT (ms) & $176 \pm 51$ & $171 \pm 53$ & $182 \pm 50$ & 0.48 \\
\hline A wave velocity $(\mathrm{cm} / \mathrm{s})$ & $41 \pm 11$ & $38 \pm 10$ & $44 \pm 12$ & 0.07 \\
\hline E/A ratio & $1.31 \pm 0.39$ & $1.43 \pm 0.35$ & $1.14 \pm 0.39$ & 0.01 \\
\hline RVOT VTI (cm) & $13 \pm 4$ & $12 \pm 4$ & $14 \pm 4$ & 0.10 \\
\hline RVOT-FS (\%) & $22 \pm 6$ & $20 \pm 6$ & $25 \pm 6$ & 0.01 \\
\hline GLS-RV (\%) & $-12.1 \pm 5.1$ & $-10.5 \pm 4.5$ & $-14.3 \pm 5.2$ & 0.009 \\
\hline RVFW-LS (\%) & $-14.8 \pm 8.3$ & $-12.9 \pm 8.7$ & $-17.5 \pm 7.1$ & 0.046 \\
\hline $\mathrm{RA}$ area $\left(\mathrm{cm}^{2}\right)$ & $15.1(13.3-19.1)$ & $15.2(13.7-22.5)$ & $15(13-16.7)$ & 0.39 \\
\hline More than mild TR, n(\%) & $11(22 \%)$ & $9(31 \%)$ & $2(10 \%)$ & 0.07 \\
\hline PASP $(m m ~ H g)$ & $39 \pm 17$ & $40 \pm 18$ & $39 \pm 19$ & 0.84 \\
\hline
\end{tabular}

Bolded p-values are statistically significant $<0.05$

Data are expressed as mean \pm standard deviation or median (interquartile range). Categorical data are expressed as number (percentage). Units of measurement are given in parentheses

$n$ number of patients, $L V$ left ventricle, $L V E D V$ LV end-diastolic volume, $L V E S V$ LV end-systolic volume, $L V E F$ LV ejection fraction, $D T$ deceleration time, $O T$ outflow tract, $V T I$ velocity time integral, $G L S$ global longitudinal strain, $L A$ left atrium, $R V$ right ventricle, $R V-F A C \mathrm{RV}$ fractional area change, TAPSE tricuspid annular plane systolic excursion, $F S$ fractional shortening, $R V F W-L S$ RV free wall longitudinal strain, $R A$ right atrium, $T R$ tricuspid regurgitation, $P A S P$ pulmonary artery systolic pressure predicted adverse cardiovascular events (Fig. 5); the difference in event-free survival was greater when stratified by TAPSE and 3D RVEF.

As shown in Tables 5, NYHA class, mitral E/E' ratio, LA volume index and most of the RV functional parameters predicted the composite end-point in univariable Cox regression analysis. Since RV functional parameters showed good correlation to one another, they were not fitted together in the same multivariable model in order to avoid multicollinearity and overfitting. By multivariable Cox regression (Table 6), TAPSE, S wave velocity, GLS-RV and 3D RVEF were significantly associated with MACE after adjustment for clinical factors (model 1), while RVFW-LS was not (HR: 1.055, 95\% CI: 0.997-1.117, p=0.063). However, 3D RVEF remained the only independent predictor of adverse events after further adjustment for mitral $\mathrm{E} / \mathrm{E}^{\prime}$ ratio (model 2 ) and subsequently also for LA volume index (model 3 ). Furthermore, RVEF remained an independent predictor of the composite endpoint in multivariable models when treated as a categorical variable, using the cut-off of $43.4 \%$ obtained from ROC analysis (Table 6). Neither LVEF nor GLS-LV 
Table 3 Baseline 3D echocardiographic data

\begin{tabular}{|c|c|c|c|c|}
\hline Variables & $\begin{array}{l}\text { All patients } \\
(\mathrm{n}=50)\end{array}$ & $\begin{array}{l}\text { MACE } \\
(n=29)\end{array}$ & $\begin{array}{l}\text { No MACE } \\
(\mathrm{n}=21)\end{array}$ & P-value \\
\hline \multicolumn{5}{|l|}{ LV parameters } \\
\hline 3D LVEDV index $\left(\mathrm{ml} / \mathrm{m}^{2}\right)$ & $127 \pm 47$ & $133 \pm 46$ & $120 \pm 49$ & 0.34 \\
\hline 3D LVESV index $\left(\mathrm{ml} / \mathrm{m}^{2}\right)$ & $96 \pm 42$ & $99 \pm 40$ & $91 \pm 44$ & 0.49 \\
\hline 3D LVEF $(\%)$ & $26 \pm 6$ & $27 \pm 7$ & $26 \pm 6$ & 0.80 \\
\hline \multicolumn{5}{|l|}{ RV parameters } \\
\hline 3D RVEDV index $\left(\mathrm{ml} / \mathrm{m}^{2}\right)$ & $75 \pm 31$ & $75 \pm 33$ & $76 \pm 30$ & 0.96 \\
\hline 3D RVESV index $\left(\mathrm{ml} / \mathrm{m}^{2}\right)$ & $43 \pm 17$ & $46 \pm 20$ & $39 \pm 13$ & 0.13 \\
\hline 3D RV stroke volume index $\left(\mathrm{ml} / \mathrm{m}^{2}\right)$ & $32 \pm 18$ & $29 \pm 15$ & $37 \pm 21$ & 0.17 \\
\hline 3D RVEF (\%) & $42 \pm 10$ & $38 \pm 8$ & $47 \pm 9$ & 0.001 \\
\hline
\end{tabular}

Bolded p-values are statistically significant $<0.05$

Data are expressed as mean \pm standard deviation. Units of measurement are given in parentheses

$L V$ left ventricle, $E D V$ end-diastolic volume, $E S V$ end-systolic volume, $E F$ ejection fraction, $R V$ right ventricle
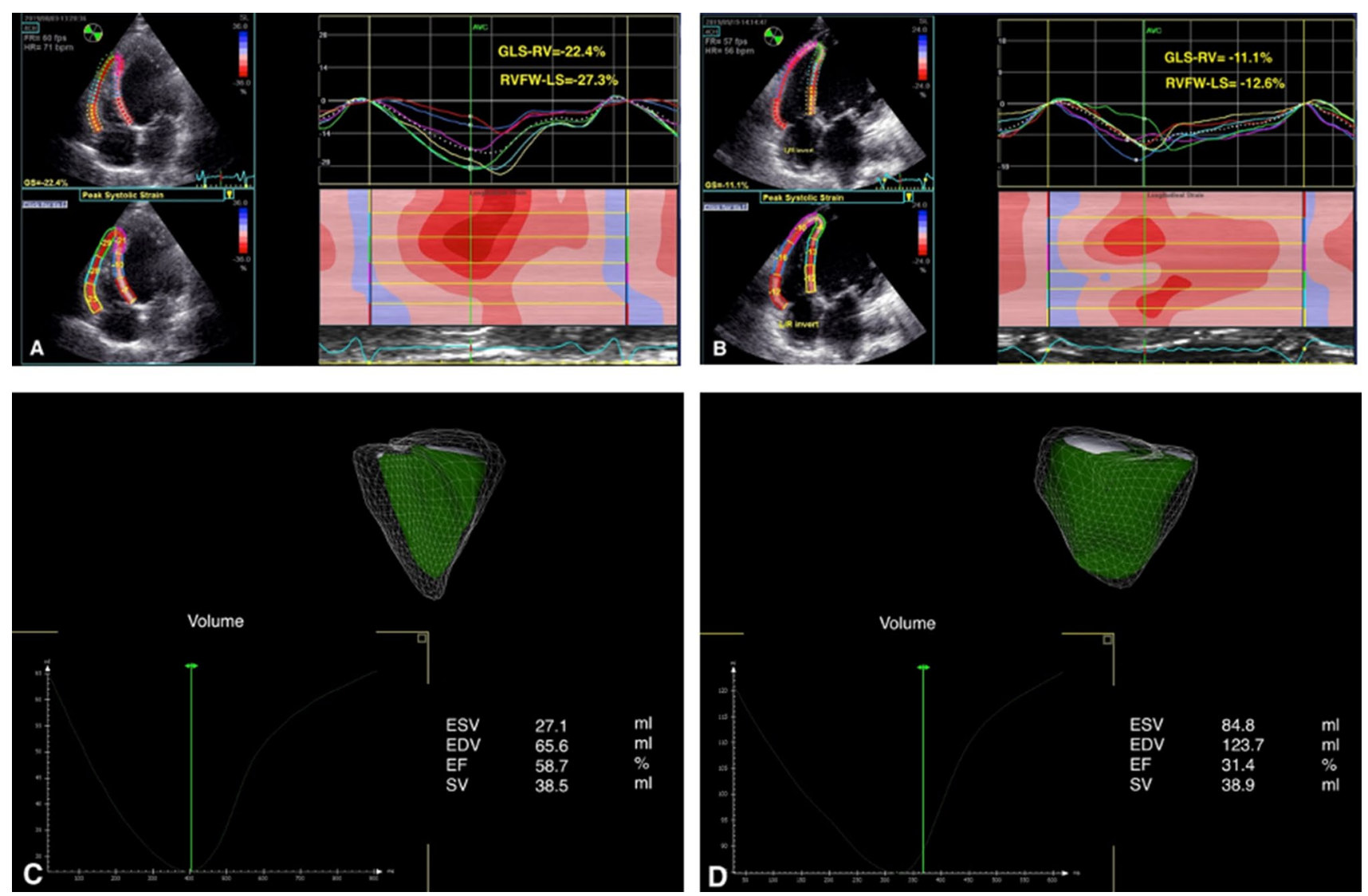

Fig. 3 Comparative analysis of RV function in two patients, one from the MACE group (panels B and D) and one from the group free of MACE (panels A and C). A Strain analysis of the RV showing normal values of both GLS-RV and RVFW-LS. B Strain analysis of the RV showing impaired values of both GLS-RV and RVFW-LS. C 3D volumetric analysis of the RV showing a preserved 3D RVEF. D 3D

volumetric analysis of the RV showing a reduced 3D RVEF. $R V$ right ventricle, MACE major adverse cardiovascular events, $G L S-R V$ global longitudinal strain of the RV, RVFW-LS RV free wall longitudinal strain, $3 D$ three dimensional, $E S V$ end-systolic volume, $E D V$ enddiastolic volume, $E F$ ejection fraction, $S V$ stroke volume 


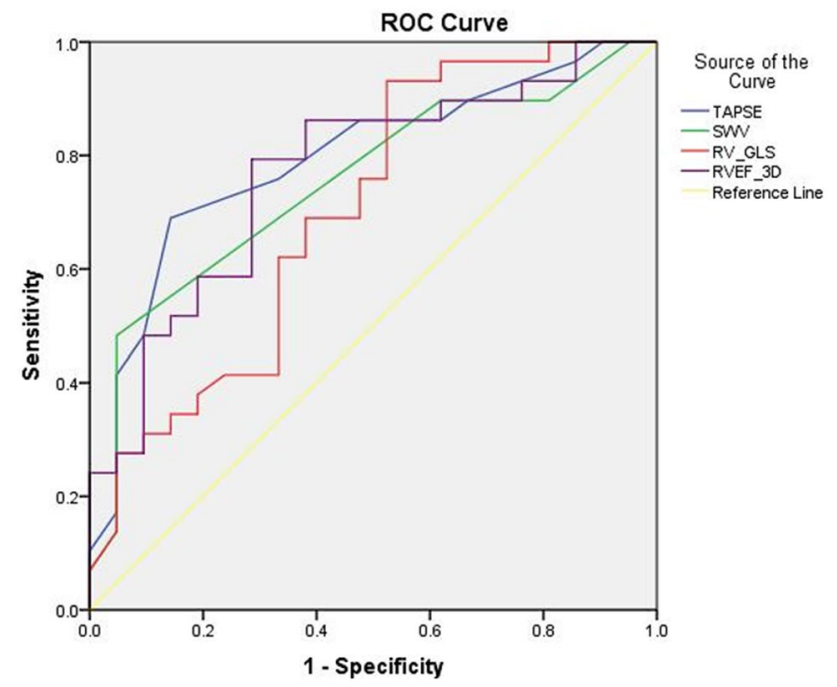

Fig. 4 Receiver-operating characteristic analysis of right ventricular functional parameters for the prediction of adverse events. TAPSE tricuspid annular plane systolic excursion, $S W V \mathrm{~S}$ wave velocity, $R V \_G L S$ right ventricular global longitudinal strain, $R V E F \_3 D$ right ventricular ejection fraction

were fitted into the multivariable models since they were not significant predictors in univariable analysis.

\section{Discussion}

The main findings of this study were: (1) there was a high prevalence of RV dysfunction among patients with DCM; (2) GLS-RV, RVFW-LS and 3D RVEF were significantly more impaired in patients with MACE; (3) GLS-RV and 3D RVEF were independent predictors of adverse outcome after adjusting for clinical risk factors; (4) 3D RVEF remained the only independent predictor of MACE after controlling for both clinical and echocardiographic variables.

It is currently acknowledged that the RV plays an important role in different cardiac diseases, with RV failure carrying significant morbidity and mortality in HF with reduced or preserved EF [18]. The echocardiographic assessment of the RV is challenging, since traditional parameters of RV function are angle- and load-dependent [14], and they neglect the complex contraction pattern of the RV [19, 20]. RV strain derived from 2D STE detects subtle myocardial abnormalities and it is relatively angle- and load-independent [21]. 3D assessment of the RV overcomes 2D geometric assumptions, by integrating the longitudinal, radial and antero-posterior components of RV contraction [22]. In our study, the use of novel techniques such as STE and 3D echocardiography allowed the reclassification of more than $30 \%$ of the patients from normal RV function (based on traditional parameters) to impaired RV function. This finding is consistent with previous data [4], thus making questionable the assessment of RV function by conventional parameters only. In our cohort, the mean values of GLS-RV and RVFW-LS were severely impaired, while mean RVEF was only mildly decreased. This suggests that longitudinal RV strain detects more subtle myocardial abnormalities than RVEF, a finding consistent with previous data [23].

In the current study, patients with GLS-RV > $-12.5 \%$ had worse outcome by Kaplan-Meier analysis and GLS-RV was significantly associated with MACE after adjustment for clinical risk factors. This highlights the importance of ventricular interdependence in patients with HF, since GLS-RV also reflects the contraction of the IVS, whose myoarchitecture has muscle fibres from both ventricles. Similar prognostic value of RV strain in patients with $\mathrm{HF}$ and reduced $\mathrm{EF}$ (HFrEF) has been previously reported [4, 24, 25]; in a recent study, Houard et al. found that RV strain is a better mortality predictor in HFrEF than conventional echocardiographic parameters, CMR-derived RVEF and CMR-derived RV strain [26]. However, in our study, GLS-RV did not remain an independent predictor of events after further adjustment for LV diastolic dysfunction. One possible explanation for this is that the LA is the pathophysiological link between LV diastolic dysfunction and its impact on the pulmonary vascular bed and the RV [27]. Significant association between LV diastolic dysfunction and impaired RV strain has been previously described [28].
Table 4 AUC and optimal cut-off value for RV functional parameters to identify patients with MACE

\begin{tabular}{llrcll}
\hline Parameter & AUC $(95 \%$ CI $)$ & P-value & Cut-off value & Sensitivity (\%) & Specificity (\%) \\
\hline TAPSE & $0.794(0.667-0.921)$ & $<0.001$ & $17.5 \mathrm{~mm}$ & $69 \%$ & 85.7 \\
S wave velocity & $0.753(0.617-0.889)$ & 0.002 & $10.5 \mathrm{~cm} / \mathrm{s}$ & 62.1 & 76.2 \\
RV-FAC & $0.614(0.454-0.775)$ & 0.172 & $35.8 \%$ & 69 & 57.1 \\
GLS-RV & $0.702(0.551-0.852)$ & 0.016 & $-12.5 \%$ & 69 & 61.9 \\
RVFW-LS & $0.634(0.479-0.788)$ & 0.109 & $-14.8 \%$ & 58.6 & 61.9 \\
3D RVEF & $0.768(0.635-0.902)$ & 0.001 & $43.4 \%$ & 79.3 & 71.4 \\
\hline
\end{tabular}

$A U C$ area under the curve, $R V$ right ventricle, $M A C E$ major adverse cardiovascular events, $C I$ confidence interval, TAPSE tricuspid annular plane systolic excursion, $R V$-FAC RV fractional area change, GLS$R V$ global longitudinal strain of the RV, RVFW-LS RV free wall longitudinal strain, $R V E F$ RV ejection fraction 
Fig. 5 Unadjusted KaplanMeier analysis for adverse events stratified by cut-off values obtained by ROC curves for tricuspid annular plane systolic excursion (upper left), right ventricular ejection fraction (upper right), $\mathrm{S}$ wave velocity (lower left) and right ventricular global longitudinal strain (lower right)
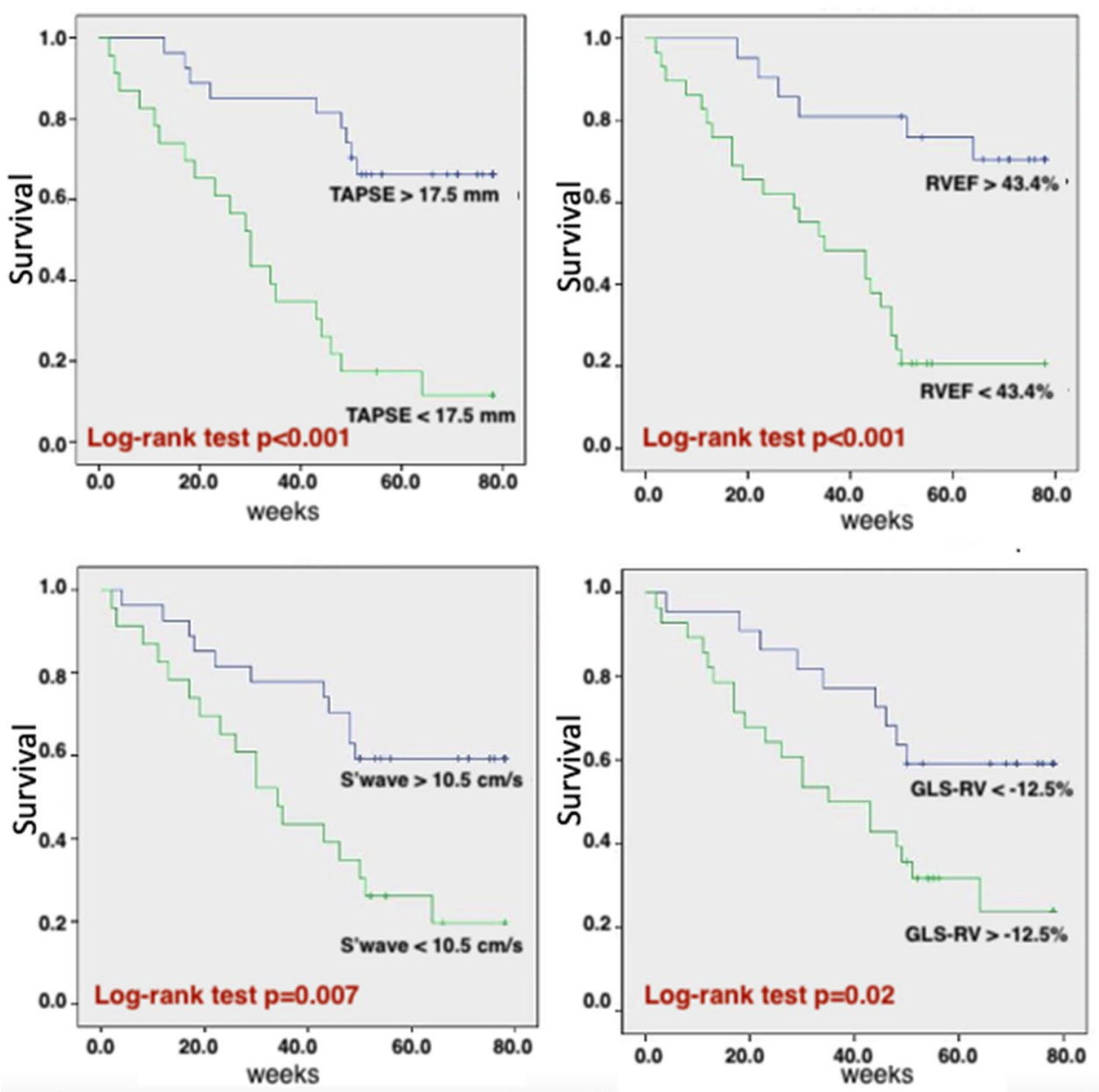

Contrary to the findings of Seo et al. [6], we found no independent prognostic value for RVFW-LS in our cohort, despite a significantly more impaired RVFW-LS in the MACE subgroup and an excellent correlation between GLSRV and RVFW-LS. A possible explanation for this might be the higher reproducibility of GLS-RV compared to RVFWLS observed in our study.

RVEF assessed by different imaging methods is a powerful predictor of outcome. A nuclear cardiology study found that RVEF $<20 \%$ is an independent predictor of death in elderly patients with HFrEF [29]. Two CMR studies in patients with DCM found that reduced RVEF is a robust predictor of adverse events [30,31]. While current guidelines recommend the $3 \mathrm{D}$ volumetric and functional evaluation of the RV when clinically important [12], the prognostic role of 3D RVEF is not thoroughly established.

Our study showed that 3D RVEF was the only independent predictor of adverse outcome, after adjusting for wellestablished clinical and echocardiographic predictors of events, such as age, NYHA class, E/E' ratio and LA volume index. A value of RVEF $<43.4 \%$ was associated with an adjusted 3-fold increased risk of MACE. RVEF and TAPSE had the highest AUC by ROC analysis for event prediction. However, except for RVEF, all the other parameters of RV function, either conventional or derived from STE-including TAPSE-lost their predictive value when fitted into the multivariable models comprising both clinical and echocardiographic factors. It is worth reminding that the contraction of the RV outflow tract (RVOT) is taken into account when measuring RVEF, but not longitudinal RV strain. The superior prognostic value of RVEF in comparison to RV strain might thus be related to the impaired antero-posterior shortening of the RV. In fact, the 2D fractional shortening of the RVOT was significantly more reduced in the MACE subgroup in our study (Table 2). A previous study found this parameter to be an independent predictor of events in HFrEF patients [32]. This highlights the importance of integrating all the components of RV contraction when assessing its systolic function.

To the best our knowledge, this is the first prospective study to assess the role of 3D RVEF in patients with DCM, and the first study to assess the prognostic value of both 3D RVEF and RV strain in the same cohort of patients with DCM. Nagata et al. evaluated the long-term 
Table 5 Univariable Cox regression analysis

\begin{tabular}{|c|c|c|}
\hline Variables & $\mathrm{HR}(95 \% \mathrm{CI})$ & P-value \\
\hline \multicolumn{3}{|l|}{ Clinical characteristics } \\
\hline Age (years) & $1.001(0.977-1.027)$ & 0.909 \\
\hline NYHA class & $2.222(1.371-3.600)$ & 0.001 \\
\hline \multicolumn{3}{|l|}{ Parameters of LV function } \\
\hline 2D LVEF & $0.970(0.921-1.020)$ & 0.237 \\
\hline GLS-LV & $1.106(0.951-1.287)$ & 0.192 \\
\hline 3D LVEF & $0.986(0.931-1.044)$ & 0.622 \\
\hline Mitral E/E' ratio & $1.101(1.042-1.164)$ & 0.001 \\
\hline LA volume index & 1.017 (1.004-1.029) & 0.007 \\
\hline \multicolumn{3}{|l|}{ Parameters of RV function } \\
\hline S wave velocity & $0.735(0.618-0.874)$ & $<0.001$ \\
\hline TAPSE & $0.833(0.760-0.912)$ & $<0.001$ \\
\hline RV-FAC & $0.974(0.945-1.003)$ & 0.079 \\
\hline GLS-RV & $1.144(1.052-1.244)$ & 0.002 \\
\hline RVFW-LS & $1.072(1.022-1.125)$ & 0.005 \\
\hline 3D RVEDV index & $1.001(0.990-1.013)$ & 0.816 \\
\hline 3D RVESV index & $1.020(1.001-1.040)$ & 0.041 \\
\hline 3D RV stroke volume index & $0.983(0.958-1.009)$ & 0.206 \\
\hline 3D RVEF & $0.915(0.873-0.960)$ & $<0.001$ \\
\hline
\end{tabular}

Bolded p-values are statistically significant $<0.05$

$h$ hazard ratio, $C I$ confidence interval, NYHA New York Heart Association, $L V$ left ventricle, $2 D$ two dimensional, $E F$ ejection fraction, $G L S$ global longitudinal strain, $3 D$ three dimensional, $L A$ left atrium, $R V$ right ventricle, TAPSE tricuspid annular plane systolic excursion, $F A C$ fractional area change, $R V F W-L S$ RV free wall longitudinal strain, $E D V$ end-diastolic volume, $E S V$ end-systolic volume. The hazard ratio refers to a unit increase in the variable analysed

prognostic value of 3D RVEF in 446 patients with various cardiovascular diseases and found that RVEF was an independent predictor of both cardiac death and of MACE [5]. However, the study was retrospective, and patients with DCM represented only $10 \%$ of the study population. A recent retrospective study evaluated the relative importance of 3D RVEF and 3D LVEF in mortality prediction among patients with different cardiovascular diseases. Impaired RVEF, but not LVEF, proved to be an independent predictor of mortality, while patients with reduced LVEF and preserved RVEF had significantly better survival than patients with reduced RVEF and preserved LVEF [33]. However, the distribution of different cardiac pathologies among the study group might have influenced the prognosis regardless of the ventricular functions.

It is worth highlighting that the primary endpoint in our cohort was mainly driven by HF readmissions, which had a $46 \%$ prevalence-slightly higher in comparison to literature data [34]. This might be explained partly by the high salt intake in our country's population [35], partly by the delay in medical check-ups and in seeking urgent care during the Coronavirus Disease 2019 (COVID-19) pandemic [36].
Table 6 Multivariable Cox regression analysis

\begin{tabular}{lll}
\hline Variables & HR $(95 \%$ CI $)$ & P-value \\
\hline GLS-RV (per unit increase) & & \\
Model 1 & $1.098(1.001-1.205)$ & $\mathbf{0 . 0 4 9}$ \\
Model 2 & $1.081(0.985-1.187)$ & 0.099 \\
Model 3 & $1.053(0.951-1.166)$ & 0.320 \\
TAPSE (per unit increase) & & \\
Model 1 & $0.870(0.776-0.975)$ & $\mathbf{0 . 0 1 6}$ \\
Model 2 & $0.891(0.790-1.006)$ & 0.062 \\
Model 3 & $0.910(0.804-1.030)$ & 0.134 \\
S wave velocity (per unit increase) & & \\
Model 1 & $0.813(0.677-0.975)$ & $\mathbf{0 . 0 2 5}$ \\
Model 2 & $0.851(0.697-1.038)$ & 0.110 \\
Model 3 & $0.892(0.725-1.096)$ & 0.276 \\
3D RVEF (per unit increase) & & \\
Model 1 & $0.928(0.881-0.976)$ & $\mathbf{0 . 0 0 4}$ \\
Model 2 & $0.926(0.878-0.976)$ & $\mathbf{0 . 0 0 5}$ \\
Model 3 & $0.926(0.876-0.980)$ & $\mathbf{0 . 0 0 7}$ \\
3D RVEF <43.4\% (yes/no) & & \\
Model 1 & $3.506(1.307-9.406)$ & $\mathbf{0 . 0 1 3}$ \\
Model 2 & $3.427(1.278-9.189)$ & $\mathbf{0 . 0 1 4}$ \\
Model 3 & $3.007(1.084-8.339)$ & $\mathbf{0 . 0 3 4}$ \\
\hline
\end{tabular}

Bolded p-values are statistically significant $<0.05$

Model 1: after adjustment for age and NYHA class

Model 2: after adjustment for age, NYHA class and mitral E/E' ratio

Model 3: after adjustment for age, NYHA class, mitral E/E' ratio and LA volume index

$H R$ hazard ratio, $C I$ confidence interval, $3 D$ three dimensional, TAPSE tricuspid annular plane systolic excursion, $R V E F$ right ventricular ejection fraction, $G L S-R V$ global longitudinal strain of the right ventricle, NYHA New York Heart Association, $L A$ left atrium

However, analysing the determinants of HF readmissions is beyond the scope of this study.

LV systolic parameters showed no prognostic value in our cohort. This happened most likely due to the narrow range of severely impaired LVEF and GLS-LV in our patients. RVEF has been previously acknowledged to attenuate the prognostic power of LVEF in multivariable models [33]. The findings of our study highlight the importance of a comprehensive RV assessment, including 3D measurements, in the evaluation of patients with DCM.

\section{Study limitations}

Our study had several limitations. First, it was a singlecentre study, with a small sample size and a relatively short follow-up period. Further studies are needed to evaluate the prognostic value of RVEF in long-term follow-up. Second, there was a risk of selection bias, as we excluded patients with poor image quality, that would have hampered accurate strain or 3D analysis. Moreover, we only included patients 
in sinus rhythm in order to avoid stitch artifacts in 3D evaluation, but atrial fibrillation is a common finding and an established prognostic marker in patients with depressed LVEF. Therefore, our results might not apply to the whole population of DCM patients. Third, reference values for RV strain from large populations with DCM are lacking. In our study, for RV strain we used vendor-specific software that was designed for the LV and adapted for the RV. The cut-off values reported in our study using ROC analysis might not apply to other software or populations. We did not include 3D STE analysis in our study. While still being limited to research and not widely available, 3D strain measurements assess the complex RV mechanics with greater accuracy [37], thus holding great promise for future research. Last, the composite endpoint was mainly driven by readmission for HF, with a small number of deaths and non-fatal cardiac arrests.

\section{Conclusions}

This study established that 3D RVEF is an independent predictor of events in patients with DCM, providing additional prognostic value over clinical risk factors and LV diastolic dysfunction. This reinforces the idea that RV function should be thoroughly evaluated in DCM, in order to improve risk stratification for these patients.

Acknowledgements This work was supported by CREDO ProjectID: 49182, financed by the National Authority of Scientific Research and Innovation, on behalf of the Romanian Ministry of European Funds- through the Sector Operational Program "Increasing of Economic Competitiveness", Priority Axis 2, Operation 2.2.1 (SOP IEC -A2-0.2.2.1-2013-1) co-financed by the European Regional Development Fund.

Author contributions Conceptualization: AV; Methodology: SO; Data acquisition: AV, CG, VV, IP, AS, SD; Data analysis and interpretation: AV, VB, DZ, RO, AS-U, RV; Writing—original draft preparation: AV; Writing-review and editing: SO, RV, MD; Supervision: MD; Final approval of manuscript: all authors.

Data availability On demand.

\section{Declarations}

Conflict of interest The authors declare that they have no conflict of interest.

Consent for participation Informed consent was obtained from all individual participants included in the study.

Consent for publication Informed consent to publish their data was obtained from all individual participants included in the study.
Ethical approval The study has been approved by the ethics committee of the Emergency Clinical Hospital Bucharest and complies with the Declaration of Helsinki.

\section{References}

1. Curtis JP, Sokol SI, Wang YW, Rathore SS, Ko DT, Jadbabaie F et al (2003) The association of left ventricular ejection fraction, mortality, and cause of death in stable outpatients with heart failure. J Am Coll Cardiol 42:736-742

2. Ponikowski P, Voors AA, Anker SD, Buneo H, Cleland JGF, Coats AJS et al (2016) 2016 ESC guidelines for the diagnosis and treatment of acute and chronic heart failure: the task force for the diagnosis and treatment of acute and chronic heart failure of the European Society of Cardiology (ESC). Eur J Heart Fail 18:891-975

3. Fine NM, Chen L, Bastiansen PM, Frantz RP, Pellikka PA, Oh JK et al (2013) Outcome prediction by quantitative right ventricular function assessment in 575 subjects evaluated for pulmonary hypertension. Circ Cardiovasc Imaging 6:711-721. https://doi. org/10.1161/CIRCIMAGING.113.000640

4. Carluccio E, Biagioli P, Alunni G, Murrone A, Zuchi C, Coiro S et al (2018) Prognostic value of right ventricular dysfunction in heart failure with reduced ejection fraction. Superiority of longitudinal strain over tricuspid annular plane systolic excursion. Circ Cardiovasc Imaging 11:e006894. https://doi.org/10.1161/CIRCI MAGING.117.006894

5. Nagata Y, Wu VC-C, Kado Y, Otani K, Lin F-C, Otsuji Y et al (2017) Prognostic value of right ventricular ejection fraction assessed by transthoracic 3D echocardiography. Circ Cardiovasc Imaging 10:e005384. https://doi.org/10.1161/CIRCIMAGING. 116.005384

6. Seo J, Jung IH, Park JH, Kim GS, Lee HY, Byun YS et al (2019) The prognostic value of $2 \mathrm{D}$ strain in assessment of the right ventricle in patients with dilated cardiomyopathy. Eur Heart J Cardiovasc Imaging 20(9):1043-1050. https://doi.org/10.1093/ehjci/ jez015

7. Zaidi A, Knight DS, Augustine DX, Harkness A, Oxborough D, Pearce K et al (2020) Echocardiographic assessment of the right heart in adults: a practical guideline from the British Society of Echocardiography. Echo Res Pract 7(1):G19-G41

8. Hundley WG, Bluemke DA, Finn JP, Flamm SD, Fogel MA, Friedrich MG et al (2010) ACCF/ACR/AHA/NASCI/SCMR 2010 expert consensus document on cardiovascular magnetic resonance: a report of the American College of Cardiology Foundation Task Force on Expert Consensus Documents. J Am Coll Cardiol 55:2614-2662

9. Park JH, Negishi K, Kwon DH, Popovic ZB, Grimm RA, Marwick TH (2014) Validation of global longitudinal strain and strain rate as reliable markers of right ventricular dysfunction: comparison with cardiac magnetic resonance and outcome. J Cardiovasc Ultrasound 22(3):113-120

10. Muraru D, Spadotto V, Cecchetto A, Romeo G, Aruta P, Ermacora D et al (2016) New speckle-tracking algorithm for right ventricular volume analysis from three-dimensional echocardiographic data sets: validation with cardiac magnetic resonance and comparison with the previous analysis tool. Eur Heart J Cardiovasc Imaging 17:1279-1289. https://doi.org/10.1093/ehjci/jev309

11. Pinto YM, Elliott PM, Arbustini E, Adler Y, Anastasakis A, Böhm M et al (2016) Proposal for a revised definition of dilated cardiomyopathy, hypokinetic non-dilated cardiomyopathy, and its implications for clinical practice: a position statement of the 
ESC Working Group on Myocardial and Pericardial Diseases. Eur Heart J 37:1850-1858

12. Lang RM, Badano PL, Mor-Avi V, Afilalo J, Armstrong A, Ernande L et al (2015) Recommendations for cardiac chamber quantification by echocardiography in adults: an update from the American Society of Echocardiography and the European Association of Cardiovascular Imaging. Eur Heart J Cardiovasc Imaging 16:233-271

13. Mitchell C, Rahko PS, Blauwet LA, Canaday B, Finstuen JA, Foster MC (2019) Guidelines for performing a comprehensive transthoracic echocardiographic examination in adults: recommendations from the American Society of Ehocardiography. J Am Soc Echocardiogr 32(1):1-64. https://doi.org/10.1016/j.echo. 2018.06.004

14. Rudski LG, Lai WW, Afilalo J, Hua L, Handschumacher MD, Chandrasekaran K et al (2010) Guidelines for the echocardiographic assessment of the right heart in adults: a report from the American Society of Echocardiography Endorsed by the European Association of Echocardiography, a Registered Branch of the European Society of Cardiology, and the Canadian Society of Echocardiography. J Am Soc Echocardiogr 23:685-713

15. Muraru D, Onciul S, Peluso D, Soriani N, Cucchini U, Aruta P et al (2016) Sex- and method-specific reference values for right ventricular strain by 2-dimensional speckle-tracking echocardiography. Circ Cardiovasc Imaging 9(2):e003866. https://doi.org/10. 1161/IRCIMAGING.115.003866

16. Badano LP, Kolias TJ, Muraru D, Abraham TP, Aurigemma G, Edvardsen T et al (2018) Standardization of left atrial, right ventricular, and right atrial deformation imaging using two-dimensional speckle-tracking echocardiography: a consensus document of the EACVI/ASE/Industry task force to standardize deformation imaging. Eur Heart J Cardiovasc Imaging 19:591-600

17. Leibundgut G, Rohner A, Grize L, Bernheim A, Kessel-Schaefer A, Bremerich J et al (2010) Dynamic assessment of right ventricular volumes and function by real-time three-dimensional echocardiography: a comparison study with magnetic resonance imaging in 100 adult patients. J Am Soc Echocardiogr 23:116-126

18. Ghio S, Guazzi M, Scardovi AB, Klersy C, Clemenza F, Carluccio E et al (2017) on behalf of all investigators. Different correlates but similar prognostic implications for right ventricular dysfunction in heart failure patients with reduced or preserved ejection fraction. Eur J Heart Fail 19(7):873-879

19. Buckberg G, Hoffman JI (2014) Right ventricular architecture responsible for mechanical performance: unifying role of ventricular septum. J Thorac Cardiovasc Surg 148(6):3166-3171

20. Kovacs A, Lakatos B, Tokodi M, Merkely B (2019) Right ventricular mechanical pattern in health and disease: beyond longitudinal shortening. Heart Fail Rev 24:511-520

21. Hernandez-Suarez DF, Lopez-Candales A (2017) Strain imaging echocardiography: what imaging cardiologists should know. Curr Cardiol Rev 13(2):118-129

22. Smolarek D, Gruchala M, Sobiczewski W (2017) Echocardiographic evaluation of right ventricular systolic function: the traditional and innovative approach. Cardiol J 24;5:563-572

23. Hirasawa K, van Rosendael PJ, Dietz MF, Marsan NA, Delgado V, Bax JJ (2020) Comparison of the usefulness of strain imaging by echocardiography versus computed tomography to detect right ventricular systolic dysfunction in patients with significant secondary tricuspid regurgitation. Am J Cardiol 134:116-122

24. Motoki H, Borowski AG, Shrestha K, Hu B, Kusunose K, Troughton RW et al (2014) Right ventricular global longitudinal strain provides prognostic value incremental to left ventricular ejection fraction in patients with heart failure. J Am Soc Echocardiogr 27(7):726-732

25. Iacoviello M, Citarelli G, Antoncecchi V, Romito R, Monitillo F, Leone $\mathrm{M}$ et al (2016) Right ventricular longitudinal strain measures independently predict chronic heart failure mortality.
Echocardiography 33(7):992-1000. https://doi.org/10.1111/echo. 13199

26. Houard L, Benaets MB, de Ravenstein CdM, Rousseau MF, Ahn SA, Amzulescu MS et al (2019) Additional prognostic value of 2D right ventricular speckle-tracking strain for prediction of survival in heart failure and reduced ejection fraction. A comparative study with cardiac magnetic resonance. JACC Cardiovasc Imaging 12(12):2373-2385

27. Marwick TH, Chandrashekhar Y (2017) The right ventricle: unforgettable with imaging. JACC Cardiovasc Imaging 10(10):1289-1290

28. Brand A, Bathe M, Oertelt-Prigione S, Seeland U, Rücke M, Regitz-Zagrosek V et al (2018) Right heart function in impaired left ventricular diastolic function: $2 \mathrm{D}$ speckle tracking echocardiography-based and doppler tissue imaging-based analysis of right atrial and ventricular function. Echocardiography 35(1):47-55. https://doi.org/10.1111/echo.13745

29. Meyer P, Desai RV, Mujib M, Feller MA, Adamopoulos C, Banach $M$ et al (2012) Right ventricular ejection fraction $<20 \%$ is an independent predictor of mortality but not of hospitalization in older systolic heart failure patients. Int J Cardiol 155(1):120-125. https://doi.org/10.1016/j.ijcard.2011.05.046

30. Gulati A, Ismail TF, Jabbour A, Alpendurada F, Guha K, Ismail NA et al (2013) The prevalence and prognostic significance of right ventricular systolic dysfunction in nonischemic dilated cardiomyopathy. Circulation 128(15):1623-1633. https://doi.org/10. 1161/CIRCULATIONAHA.113.002518

31. Doesch C, Dierks DM, Haghi D, Schimpf R, Kuschyk J, Suselbeck $T$ et al (2014) Right ventricular dysfunction, late gadolinium enhancement and female gender predict poor outcome in patients with dilated cardiomyopathy. Int J Cardiol 177:429-435. https:// doi.org/10.1016/j.ijcard.2014.09.004

32. Yamaguchi M, Tsuruda T, Watanabe Y, Onitsuka H, Furukawa K, Ideguchi T et al (2013) Reduced fractional shortening of right ventricular outflow tract is associated with adverse outcomes in patients with left ventricular dysfunction. Cardiovascular Ultrasound 11:19. https://doi.org/10.1186/1476-7120-11-19

33. Surkova E, Muraru D, Genovese D, Aruta P, Palermo C, Badano LP (2019) Relative prognostic importance of left and right ventricular ejection fraction in patients with cardiac diseases. J Am Soc Echocardiogr 32(11):1407-1415.e3. https://doi.org/10.1016/j. echo.2019.06.009

34. Wideqvist M, Cui X, Magnusson C, Schaufelberger M, Fu M (2021) Hospital readmissions of patients with heart failure from real world: timing and associated risk factors. ESC Heart Failure 8:1388-1397

35. Dorobantu M, Tautu OF, Dimulescu D, Sinescu C, GusbethTatomir P, Arsenescu-Georgescu C, Mitu F, Lighezan D, Pop C, Babes K, Giuca A, Branza I, Udrescu M, Herdea V, Darabont R (2018) Perspectives on hypertension's prevalence, treatment and control in a high cardiovascular risk East European Country: data from the SEPHAR III survey. J Hypertens 36(3):690-700

36. Wagner JR, Ambrosy AP (2020) Hospitalizations for heart failure and mortality risk during the evolving coronavirus disease 2019 pandemic - the wave may break but a dangerous undertow persists. Eur J Heart Fail 22(12):2225-2227

37. Smith BCF, Dobson G, Dawson D, Charalampopoulos A, Grapsa J, Nihoyannopoulos P (2014) Three-dimensional speckle tracking of the right ventricle - toward optimal quantification of right ventricular dysfunction in pulmonary hypertension. J Am Coll Cardiol 64:41-51

Publisher's Note Springer Nature remains neutral with regard to jurisdictional claims in published maps and institutional affiliations. 